\title{
Toi Même, a Mobile Health Platform for Measuring Bipolar Illness Activity: Protocol for a Feasibility Study
}

Aroldo A Dargél ${ }^{1,2,3}, \mathrm{MD}, \mathrm{PhD}$; Elise Mosconi ${ }^{3}, \mathrm{MD}, \mathrm{MSc}$; Marc Masson ${ }^{4}, \mathrm{MD}, \mathrm{PhD}$; Marion Plaze ${ }^{5}, \mathrm{MD}, \mathrm{PhD}$; Fabien Taieb ${ }^{6}$, MD, PhD; Cassandra Von Platen ${ }^{6}$, BSc; Tan Phuc Buivan ${ }^{6}$, BSc; Guillaume Pouleriguen ${ }^{7}$, BSc; Marie Sanchez ${ }^{7}, \mathrm{BSc}$; Stéphane Fournier ${ }^{7}, \mathrm{BSc}$; Pierre-Marie Lledo ${ }^{1,2}$, PhD; Chantal Henry ${ }^{1,5}, \mathrm{MD}, \mathrm{PhD}$

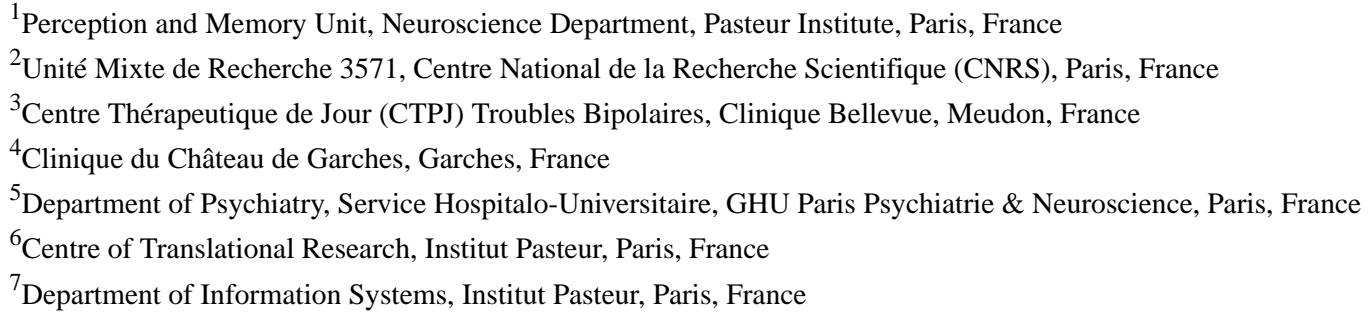

\section{Corresponding Author:}

Aroldo A Dargél, MD, PhD

Perception and Memory Unit, Neuroscience Department, Pasteur Institute

25 rue du Docteur Roux

Paris, 75015

France

Phone: 33646924206

Email: aroldo.dargel@pasteur.fr

\begin{abstract}
Background: The diagnosis and management of bipolar disorder are limited by the absence of available biomarkers. Patients with bipolar disorder frequently present with mood instability even during remission, which is likely associated with the risk of relapse, impaired functioning, and suicidal behavior, indicating that the illness is active.

Objective: This research protocol aimed to investigate the correlations between clinically rated mood symptoms and mood/behavioral data automatically collected using the Toi Même app in patients with bipolar disorder presenting with different mood episodes. This study also aimed to assess the feasibility of this app for self-monitoring subjective and objective mood/behavior parameters in those patients.

Methods: This open-label, nonrandomized trial will enroll 93 (31 depressive, 31 euthymic, and 31 hypomanic) adults diagnosed with bipolar disorder type I/II (Diagnostic and Statistical Manual of Mental Disorders, 5th edition criteria) and owning an iPhone. Clinical evaluations will be performed by psychiatrists at the baseline and after 2 weeks, 1 month, 2 months, and 3 months during the follow-up. Rather than only accessing the daily mood symptoms, the Toi Même app also integrates ecological momentary assessments through 2 gamified tests to assess cognition speed (QUiCKBRAIN) and affective responses (PLAY $i$ MOTIONS) in real-life contexts, continuously measures daily motor activities (eg, number of steps, distance) using the smartphone's motion sensors, and performs a comprehensive weekly assessment.

Results: Recruitment began in April 2018 and the completion of the study is estimated to be in December 2021. As of April 2019, 25 participants were enrolled in the study. The first results are expected to be submitted for publication in 2020 . This project has been funded by the Perception and Memory Unit of the Pasteur Institute (Paris) and it has received the final ethical/research approvals in April 2018 (ID-RCB: 2017-A02450-53).

Conclusions: Our results will add to the evidence of exploring other alternatives toward a more integrated approach in the management of bipolar disorder, including digital phenotyping, to develop an ethical and clinically meaningful framework for investigating, diagnosing, and treating individuals at risk of developing bipolar disorder or currently experiencing bipolar disorder. Further prospective studies on the validity of automatically generated smartphone data are needed for better understanding the longitudinal pattern of mood instability in bipolar disorder as well as to establish the reliability, efficacy, and cost-effectiveness of such an app intervention for patients with bipolar disorder.
\end{abstract}


Trial Registration: ClinicalTrials.gov NCT03508427; https://clinicaltrials.gov/ct2/show/NCT03508427

International Registered Report Identifier (IRRID): DERR1-10.2196/18818

(JMIR Res Protoc 2020;9(8):e18818) doi: 10.2196/18818

\section{KEYWORDS}

bipolar disorder; digital phenotyping, smartphone app; ecological momentary assessment; mHealth; mood instability; cognitive speed; affective response; big data, machine learning

\section{Introduction}

\section{Background}

In clinical practice, the diagnosis and management of $\mathrm{mood} / \mathrm{behavioral} \mathrm{symptoms} \mathrm{in} \mathrm{bipolar} \mathrm{disorder} \mathrm{rely} \mathrm{on} \mathrm{subjective}$ information and clinician's evaluations, thereby raising issues, including patient recall bias, decreased illness insight during acute affective episodes, and differences in clinical assessment experience [1]. In patients with bipolar disorder, mood changes are often accompanied by shifts in other behavioral patterns such as motor activity, energy, sleep, and cognitive functions [2]. In addition, many patients with bipolar disorder experience significant daily or weekly mood swings, which do not fulfill the criteria of an acute episode but are above the levels of $\mathrm{mood} /$ behavioral changes experienced by nonpsychiatrically ill individuals $[3,4]$.

Mood instability in bipolar disorder increases the risk for relapse [5] and impairs daily functioning over time [6-8], indicating that the illness is still active. Evidence has shown that patients with bipolar disorder in remission who presented with emotional hyper-reactivity, which was assessed as a proxy of mood instability by using an analog self-rated scale, had significantly increased risk for cardiometabolic dysfunction and poor cognitive functioning [9,10]. A recent study using a smartphone-based mood self-monitoring in patients with bipolar disorder has shown that mood instability was associated with significantly increased perceived stress, decreased quality of life, and impaired functioning, although most of these patients were in remission during the 9-month study period [11]. These findings highlight that mood instability could provide unique additional variance in predicting bipolar illness activity. However, the longitudinal pattern of mood instability is poorly understood as it is difficult to assess validly $[12,13]$. Therefore, the ability to assess mood/behavior changes continuously in real time and in more ecological conditions may be an opportunity for better understanding the clinical progression of bipolar disorder and for monitoring individual treatment outcomes.

\section{Digital Phenotyping in Bipolar Disorder}

Currently, more than $35 \%$ of the world's adult population owns and uses a smartphone [14]. Smartphones offer the opportunity to collect a vast amount of objective, fine-grained information (eg, data on phone usage, voice features, GPS data) in real time, which may reflect behavioral patterns, thereby providing novel insights into physical and mental illnesses [15-17]. As stated by the World Health Organization, "the use of mobile and wireless technologies to support the achievement of health objectives (mobile health [mHealth]) has the potential to transform health service delivery across the globe" [18]. Different mHealth interventions have been developed and used for various medical conditions such as diabetes, cardiovascular disease, asthma, and headache [19]. In psychiatry, mHealth systems for depression, anxiety, eating disorders, schizophrenia, and bipolar disorder have been gaining traction [20-26].

Several studies have evaluated the possibility of providing remote mood monitoring for patients with bipolar disorder by using diverse digital technologies [27-37]. For example, the ChronoRecord study included daily mood charting by using a computer [36,37]. In the AMoSS (Automated Monitoring of Symptoms Severity) study, participants monitored their moods daily by using a study-specific smartphone app, and they completed their weekly mood measures by using the True Colours system [38] and by wearing movement-sensing devices to monitor multiple physiological parameters [37]. This study has demonstrated that mood and activity monitoring were well accepted and tolerated by the participants who also reported that mood monitoring assisted them in the early recognition of their mood states [34]. The MONARCA (MONitoring, treAtment and pRediCtion of bipolAr Disorder Episodes) studies showed correlations between the severity of depressive and manic symptoms self-reported by patients with bipolar disorder who were using an electronic device and the clinically rated symptoms measured using standard mood rating scales [16]. The OpenSIMPLe feasibility study, which used a smartphone-based psychoeducation program for bipolar disorder, reported high percentages of perceived helpfulness, well-being, and general health among all the participants [33]. Moodswings, an internet-based self-help program for bipolar disorder, which includes psychoeducational material and cognitive behavioral therapy elements, has reported significant reductions in mood symptoms and improvements in the quality of life and medication adherence in patients with bipolar disorder who were using this platform [27].

Despite the fast growing development of digital technologies and the excessive hype for their use in psychiatry, robust evidences in this emerging field are lacking. For example, compelling pilot results of many app studies have not translated into clinical practice $[35,39]$. Standardized methods to collect, analyze, and report digital mood/behavioral data as well as clear frameworks regarding privacy and security of data are still not available [40,41], making the implementation of feasibility and validation studies an important step of this process.

\section{Objectives of This Study}

In order to tackle some of these questions as well as to contribute to the evidence on digital phenotyping in bipolar disorder, we developed the Toi Même mHealth platform, which comprises 
the Toi Même smartphone app to self-monitor subjective and objective parameters of bipolar illness activity. The name of this project was inspired by the foreword engraved on the frontispiece of the Temple of Delphi- "Know thyself" (ie, Connais toi toi-même, in French). This expression was adopted by the philosopher Socrates who uttered it in his dialogues with his mentor Platon: "Know thyself as the dweller of the mind, senses, and the body" [42]. Our research protocol was carried out in real-world clinical settings with patients with bipolar disorder and we aimed to investigate the correlations between the clinically rated mood symptoms and mood/behavioral data that were automatically collected using the Toi Même app in patients with bipolar disorder presenting with different mood episodes. This study also aimed to assess the feasibility of this app for self-monitoring subjective and objective mood/behavior parameters in these patients.

\section{Methods}

\section{Study Design}

This is an applied research study on digital technology, software development, and analysis of app feasibility through an open-label, prospective, and multicenter clinical trial. The 3 investigation centers involved in this study are The Therapeutic Center for Bipolar Disorder (Centre Thérapeutique de Jour-Troubles Bipolaires, CTPJ-TB), Clinique Bellevue, Meudon, France; Clinique du Château de Garches, Garches, France; and the Service Hospitalo-Universitaire GHU Sainte-Anne, Paris, France.

\section{Study Population}

The enrollment of the patients started in April 2018 at the CTPJ-TB investigation center. The inclusion criteria for this study were male or female adult patients with bipolar disorder (age, 18 years and above), diagnosed with bipolar disorder type I or II (Diagnostic and Statistical Manual of Mental Disorders, 5th edition [DSM-5] criteria), owning an iPhone (iPhone operating system 9.0 or higher) with wireless internet access, and with a sufficient level of understanding to follow the research protocol. All patients were assessed using a standardized semistructured clinical interview and self-reported questionnaires conducted by a trained psychiatrist. The exclusion criteria were the current DSM-5 diagnosis of schizophrenia, psychotic disorders, dementia, or mental retardation, and patients presenting with suicidal behavior/ideation. At the CTPJ-TB, an independent psychiatrist researcher (AAD) asked the patients if they would like to participate in the study and explained the aims of the study. If the patient agreed to participate, an informed consent form was handed out and signed by both the participant and the psychiatrist investigator (EM) who was blinded to the smartphone data. No rewards or incentives were offered to the patients for participating in the study, and all interviews and follow-up assessments were carried out by the same investigator. The Research Ethics Committee (Comité de Protection des Personnes, Ile-de-France VII) approved the study protocol ID-RCB: 2017-A02450-53, which is also registered at www.clinicaltrials.gov (Identifier: NCT03508427).

\section{Clinical Assessments}

Data on sociodemographic and clinical assessments were performed at baseline and after 2 weeks, 1 month, 2 months, and 3 months. These are the time points at which clinical evaluation is routinely performed at the CTPJ-TB. Hence, the study assessments did not add any extra burden on the participants or extra contact time with the staff.

The severity of the depressive and manic symptoms was evaluated using the Montgomery-Åsberg Depression Rating Scale (MADRS) [43], a 10-item questionnaire, ranging from 0 to 60 , with higher scores indicating more severe depression, and the Young Mania Rating Scale (YMRS) [44], a 11-item questionnaire, ranging from 0 to 60 , with higher scores indicating severity of (hypo)manic symptoms. Overall functioning was assessed using the Functioning Assessment Short Test (FAST), which encompasses 24 items to evaluate 6 functional domains: autonomy, occupational functioning, financial issues, interpersonal relationships, leisure time, and cognitive functioning. FAST scores range from 0 to 72 , and higher scores indicate poorer functioning and greater disability [8].

In addition to the clinical evaluations, participants completed self-report evaluations of mood symptoms, behavior, and sleep patterns by using validated instruments. Depressive and manic symptoms were self-assessed using the 16-item Quick Inventory of Depressive Symptomatology Self-Report (QIDS-SR) [45] and the Altman Self-Rating Mania, a 5-item scale [46], respectively. Sleep patterns were assessed using the Pittsburgh Sleep Quality Index [47], which differentiates "poor" from "good" sleep by assessing 7 sleep domains, that is, quality, latency, duration, habitual sleep efficiency, sleep disturbances, use of sleep medication, and daytime dysfunction. A global score of 5 or greater indicates sleep disturbances. Levels of activation were measured using the Multidimensional Assessment of Thymic States, a 20-item self-report instrument that assesses the levels of activation uncoupled from mood during the preceding week [48]. It quantitatively evaluates 5 dimensions, namely, emotional reactivity, sensory perception, psychomotor activity, motivation, and cognition, each of which can vary from hypoactivation to hyperactivation [7]. At the end of each evaluation, the investigators asked patients to give feedback regarding the overall satisfaction, ease of use, and perceived helpfulness of the Toi Même app (ranging from 1=not at all to $5=$ extremely).

The clinical data were collected using the Research Electronic Data Capture (REDCap) software [49], which is a widely used data collection platform. REDCap follows the international rules for validation, qualification, and security gold standards, which are also aligned with the Pasteur Institute's privacy/security rules for data collection.

\section{Toi Même App}

Rather than only accessing the categories of mood symptoms, the Toi Même app expands on the existing digital self-assessments for bipolar disorder by integrating ecological momentary assessments of the fundamental dimensions of behaviors (eg, motor activity, cognition, affective response) in 
the same tool through 2 gamified tests, which were created and adapted by the first author (AAD) in order to assess cognition speed (QUiCKBRAIN) and affective responses (PLAYiMOTIONS) in real-life contexts. These games also passively collect data regarding user's response time (seconds) and the number of hits/errors in each game trial, continuously measure the daily motor activities (eg, number of steps, distance) using the smartphone's motion sensors, and perform a comprehensive weekly assessment, including validated self-rating scales $[45,46,48]$. Moreover, this app is special because Toi Même is the first app developed in France without commercial purposes, and it has been developed specifically for monitoring bipolar illness activity.

The principles, content, and the specifications of the Toi Même app were conceived by AAD. After a year of collaborative work among psychiatrists, neuroscientists, software engineers, and designers, the Toi Même app 1.0 version used in this study was available in French and was free of charge for the study participants. The information technology department of Pasteur Institute provided all the technical support for coding the app and the platform's back office. The Toi Même app functionalities are intended to be minimally invasive to the users' daily routine and normal smartphone usage. After the patients were included in the study, patients received an email with a link to install the Toi Même app in their own smartphones. Once the app was installed, users could configure it to receive a notification to perform their daily/weekly assessments. The system automatically sends a reminder notification once if the users have not completed their assessments. The app allows collecting $\mathrm{mood} / \mathrm{b}$ havior information in both active (ie, need the user's action) and passive ways (ie, without the user's action), which is captured using the smartphone sensors. Once a day, the app prompts the user to score a short graphic 8-item test, including the assessment of subjective levels of mood, energy, emotion, irritability, anxiety, motor activity, speech, and thought speed on a scale from -3 to +3 . Sleep time (hours), daily events (yes/no), and medication intake (yes/no) are also assessed daily. The app also randomly delivers to the users each one of the games twice a week.

The game QUiCKBRAIN randomly offers a series of 7 trials, each one containing a pair of word image or a simple calculation. The user's action is to analyze and answer if the word matches (or not) with the image (Figure 1A) or in case a calculation is presented, the user must answer if the result of the calculation is correct (or not) (Figure 1B). Correct and incorrect buttons are present in each trial. The QUiCKBRAIN task contains about 1000 different trials.

The game PLAY $i$ MOTIONS is intended to assess the user's affective response to the images. This test comprises about 1000 color images depicting a broad spectrum of themes, including humans, animals, objects, and scenes along with normative ratings on the affective/valence dimension (ie, the degree of positive, neutral, or negative affective response that the image evokes). In each game trial, an image appears on the smartphone screen for approximately 5 seconds (Figure 2A). Thereafter, the image fades away, after which a colored circle and 2 buttons appear: one button contains the name of the color of the circle written in the same color (or in a different color) as that of the circle and another button contains the name of a color different from that of the color of the circle. In this task, the user's action is to analyze the 2 options offered and choose the one that corresponds with the color of the circle displayed on the screen (Figure 2B).

At the end of the daily assessment, the Toi Même app provides an intuitive dashboard reflecting some of the daily scores, the user's mood/behavioral patterns during the last 7 days and, by data push, during the last 30 days (Figure 3). No diagnostic or therapeutic feedbacks are provided. All data recorded by the app are encrypted, synchronized, and stored in a deidentified format within the Toi Même server. 
Figure 1. Example of a cognitive speed assessment by the QUiCKBRAIN task.

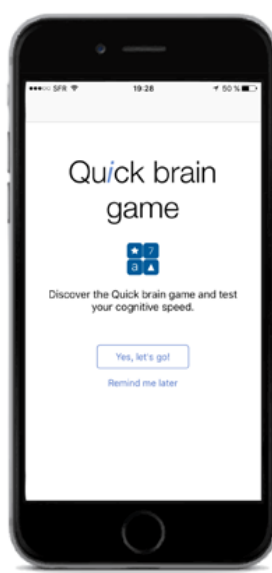

A

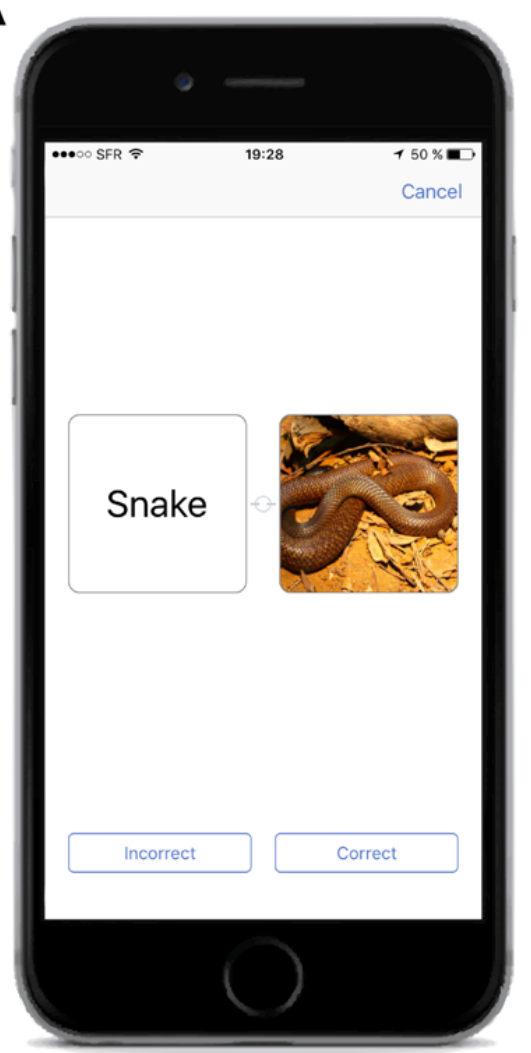

$\mathrm{B}$

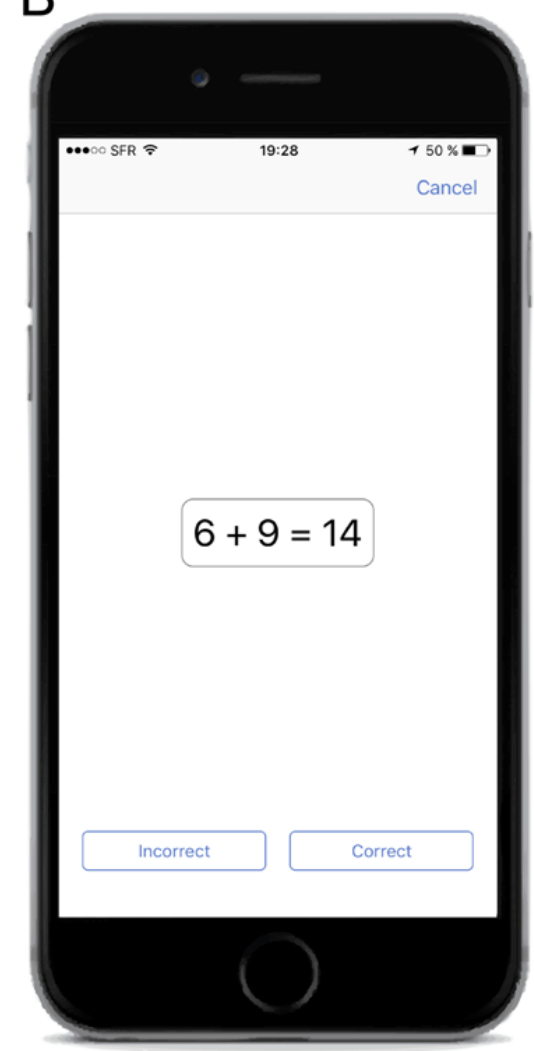


Figure 2. Example of an affective response assessment by the PLAY $i$ MOTIONS task.

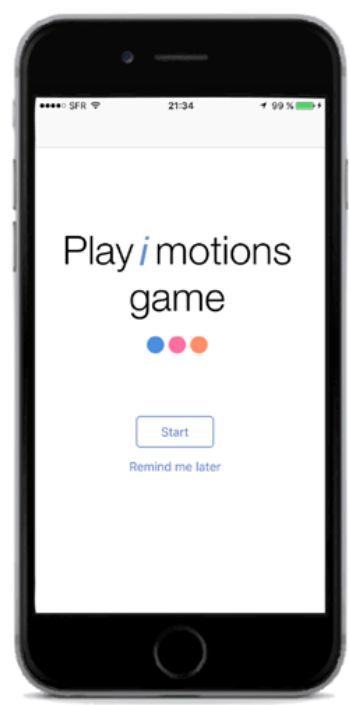

A

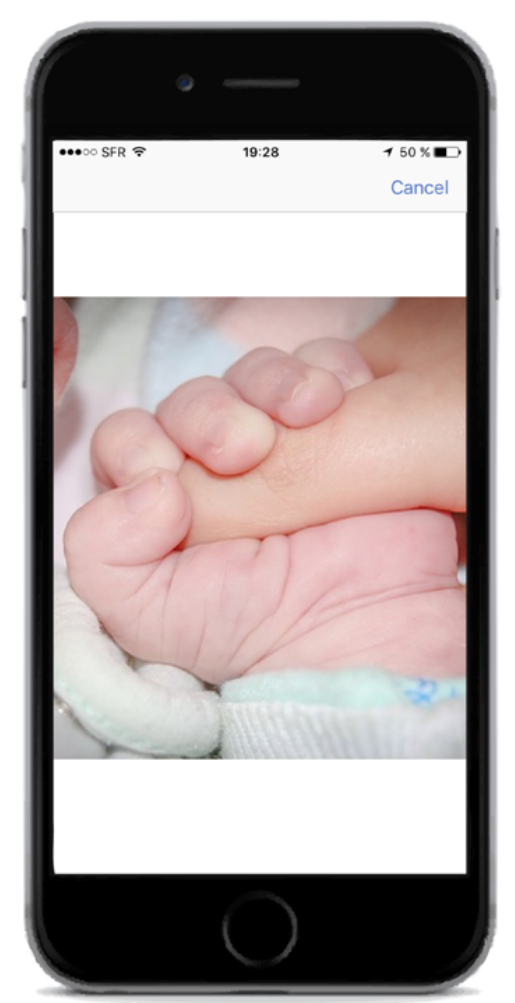

B

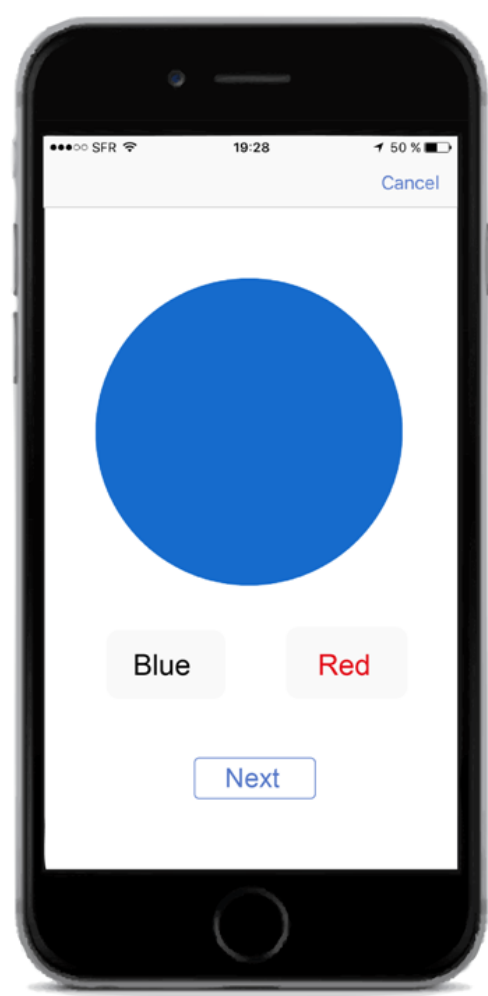


Figure 3. Example of the Toi Même app dashboard.
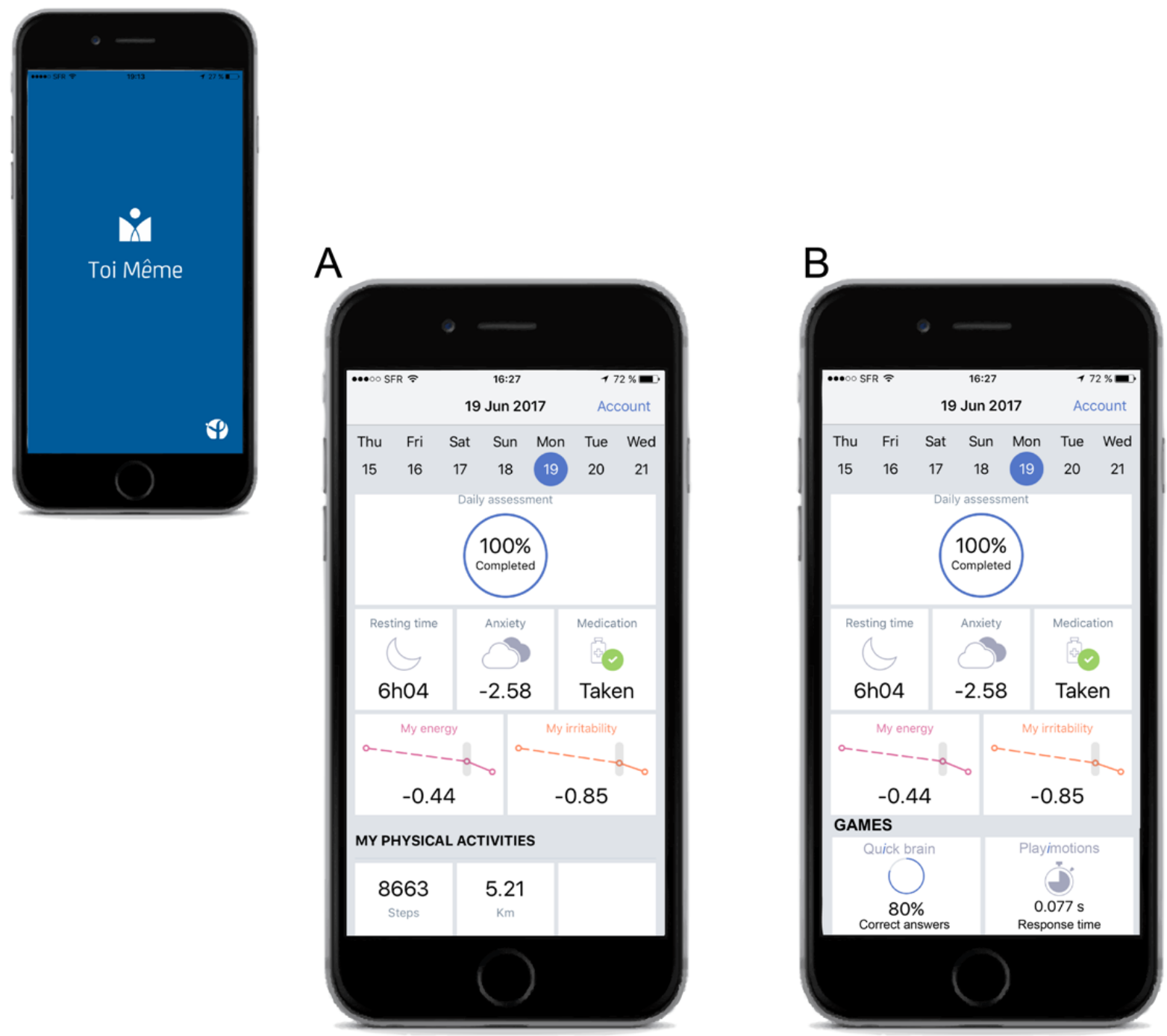

\section{Outcome Measures}

Outcome measures are assessed at the baseline visit and after 2 weeks, 1 month, 2 months, and 3 months [50]. Briefly, primary outcome measures focus on changes in the severity of the depressive and manic symptoms assessed using the MADRS and YMRS. Secondary outcomes include changes in the self-rated mood/behavioral symptoms and medication adherence assessed using the self-report instruments QIDS-SR, Altman Self-Rating Mania, Multidimensional Assessment of Thymic States, and the Medication Adherence Rating Scale [51]. Functioning will be assessed using the FAST scale. Feasibility measures include the proportion of eligible participants who have consented to participate in the study, the frequency of self-assessments, and the proportion of participants who continued using Toi Même app during the study period. The participant's physical activity will be inferred using the number of steps and distance automatically captured by the smartphone motion sensors.

\section{Sample Size}

Considering that the focus of this pilot study is to have patients with bipolar disorder in different mood states (depression, euthymia, and hypomania), a conservative approach for calculating the sample size was adopted (ie, concordant pairs) to ensure more qualitative feedback [52]. The overall $\mathrm{mood} / \mathrm{behavior}$ states assessed using the Toi Même app encompasses 8 dimensions of behavior (ie, mood, energy, emotion, irritability, anxiety, activity, speech, and thought speed). Each of these dimensions is rated using a score ranging from -3 to +3 , yielding a total score of 24 . Accordingly, a concordant pair between the app measurements and the clinical assessments of mood symptoms was defined as follows: (1) depression, a score of $<-8$ in the app assessments and scores of $>15$ for MADRS and $<8$ for YMRS; (2) euthymia, a score between -8 and +8 in the app assessments and scores of $<15$ for MADRS and $<8$ for YMRS; (3) (hypo)mania, a score of $>+8$ in the app and scores of $<15$ for MADRS and $>8$ for YMRS. Assuming that the expected proportion of the concordant pairs is $98 \%$, with an estimated precision of 5\% and a two-sided $95 \%$ CI, 31 patients will be included in each of the 3 groups to fully accomplish the study goals $(\mathrm{N}=93)$. 


\section{Statistical Analysis}

Descriptive analyses will be conducted to characterize the sociodemographic and clinical characteristics of the initial sample as well as the retention at the end of the study. Receiver operating characteristics analysis will be applied to calculate the concordance ratio of the pairs (ie, the area under the curve) [53]. Given that patients have started using the app from the baseline visit, the clinical data collected at this time point will not be included in the concordant pairs' analysis. Compliance will be calculated as the proportion of the enrolled patients completing the first 15 and then 30 and 90 consecutive days of ratings. The app completion rate would be completing at least $70 \%$ of the momentary assessments during the time a participant has continued in the study. The average ratings of the perceived helpfulness, ease of use, and overall satisfaction would be at least 3 on the 1 to 5 rating scores. Cluster analysis will be performed using principal component analysis (PCA) to identify groupings of the daily Toi Même mood/behavior questions. The PCA results will be then used to obtain a single summary statistic (TMP1) that will account for the maximum amount of variability in the 8 daily Toi Même mood/behavior responses across all the subjects during the study period. The summary statistic produced from the PCA-TMP1 will be used as a response to test for differences between groups in daily ratings and for comparison between this app score and the clinical rating scores. To test for differences between groups over time, linear mixed regression models will be used with autoregressive covariance adjustments for repeated measurements of continuous outcomes and logistic generalized estimating equations will be used for binary outcomes. In both linear and logistic generalized estimating equation models, adjustments for sex, age, day of the week, and elapsed time in the study will be included as other covariates in the models. The regularity in the daily ratings for each subject will be estimated using the root mean squared successive differences (a measure of the variability of the changes in scores over time), the Teager-Kaiser Energy Operator, which combines amplitude and frequency, and the information entropy, which measures regularity of amplitude [37]. The within-subject sample variance will be also used as a measure of regularity for each subject's response and will estimate the distribution of the amplitudes recorded over time. Data analysis will employ the intention-to-treat principle by including all participants in the analyses, which will be conducted using the $\mathrm{R}$ programming language ( $\mathrm{R}$ Core Team).

\section{Results}

The recruitment of patients started in April 2018 and the completion of the study is estimated to be in December 2021. As of April 2019, 25 participants were enrolled in the study. The first results are expected to be submitted for publication in 2020. The association between performance on both the QUiCKBRAIN and PLAYiMOTIONS and subjective cognitive/emotional functioning difficulties will be evaluated in a subsequent specific study. In the future, the transfer of technology from the academic environment to the clinics may be a strategy to invigorate efforts, making available the $T o i$ Même mHealth platform for individuals at risk of developing bipolar disorder or for those experiencing bipolar disorder. This project has been funded by the Perception and Memory Unit of the Pasteur Institute (Paris) and it has received the final ethical/research approvals in April 2018 (ID-RCB: 2017-A02450-53).

\section{Discussion}

To our knowledge, this is the first study in France that has investigated correlations between clinically rated mood symptoms and mood/behavioral data automatically collected using a smartphone app specifically developed for patients with bipolar disorder through a registered clinical trial carried out in real-world bipolar disorder treatment clinics. This multidimensional digital phenotyping approach may help to improve our understanding of bipolar illness activity and potentially contribute to early detection of subtle mood/behavior changes and to objective assessment of the response to treatment and its impact on mood, cognition, emotion, and motivation in patients with bipolar disorder. In addition, the use of digital technology in bipolar disorder treatment clinics may encourage patients with bipolar disorder to monitor their health as well as reinforce the patient-clinician therapeutic alliance.

Although the Toi Même project is not the first to implement a smartphone-based self-monitoring tool for patients with bipolar disorder, this app was designed as an independent self-assessment tool targeting mainly the fundamental dimensions of behavior (eg, activity, energy, cognition speed, affective responses) rather than as an augmenting intervention measuring essentially mood symptoms. The Toi Même clinical trial has carefully followed the ethical and legal issues around providing and obtaining adequate informed content from participants, personal privacy, and safety of data collection/storage. The same clinical psychiatrist who was blinded to smartphone data collected during the study assessed the patients by using standardized rating scales. The Toi Même app automatically integrates behavior data collection (eg, physical activity, user's response time) and 2 gamified modules targeting cognitive speed (QUiCKBRAIN) and affective response to images (PLAY $i$ MOTIONS).

Smartphones are able to automatically collect a large amount of complex and diverse data that are quickly generated and could represent a paradigm shift for accessing mood/behavior in patients with bipolar disorder, thereby providing opportunities for investigation and hypothesis generation [54,55] as well as for enhancing the precision of clinical algorithms, which today are essentially based on patients' subjective self-report and clinicians' observations. Promising progress in big data analytics (eg, machine learning techniques) as well as intensive multidisciplinary collaboration, including clinicians, neuroscientists, engineers, and data scientists, could further help to solve this complex puzzle, extracting meaningful indicators of bipolar disorder onset, course, and treatment response [56,57]. Future studies investigating the use of combined automatically generated smartphone data with biological and clinical measures may lead to the discovery and validation of digital markers of risk, staging, treatment response, and prognosis in patients with bipolar disorder [58]. 
Owing to resource constraints, this research protocol has included a Toi Même app version using only the iPhone operating system; hence, Google's Android users as well those using smartphones with other types of operating systems were excluded. This could represent a sampling bias since smartphone ownership could be related to income status, education levels, and gender [14]. Three months of follow-up may be a short time frame to evaluate the feasibility of a digital self-monitoring tool for patients with bipolar disorder [39]. However, to date, there is no consensus on how long these types of interventions should be offered. Moreover, there is a multitude of smartphones in the market in which are embedded diverse sensors as well as different operating systems, which have different permissions rules to capture passive information (eg, the iPhone operating system has more restrictions than the Android operating system). To overcome this issue, research groups using digital phenotyping in psychiatry should keep trying to communicate with information technology companies regarding the development of clear and ethical frameworks for obtaining access to more homogeneous automatically generated smartphone data in order to evaluate their validity, sensitivity, and specificity for monitoring bipolar illness activity.

In summary, the Toi Même study is an example of the use of digital phenotyping in bipolar disorder clinical practice that can provide fine-grained mood and behavioral patterns of bipolar illness activity in real time. This clinical neuroscience-based research may add to the evidence of exploring other alternatives toward a more integrated approach, including digital phenotyping, in the management of bipolar disorder to develop a clinically meaningful framework for investigating, diagnosing, and treating individuals at risk of developing bipolar disorder or currently experiencing bipolar disorder.

\section{Acknowledgments}

This work was supported by the Perception and Memory Unit and the Department of Information Systems of the Pasteur Institute (Paris). AAD is supported by a fellowship grant from the Laboratory of Excellence Program 'Revive' and the life insurance company AG2R La Mondiale. The funding agencies had no role in the conduct or publication of the study. We thank both the Departments of IT and of Legal/Industrial Affaires at the Pasteur Institute for their technical and administrative support. We also thank the patients and the staff of the Centre Thérapeutique de Jour Troubles Bipolaires (Clinique Bellevue) for their contributions.

\section{Authors' Contributions}

AAD conceived the concept, content, and the specifications for the Toi Même app. AAD, CH, and PML worked with both the IT Department and the Translational Research Center at the Pasteur Institute to develop this project. AAD drafted the protocol, and all the authors revised and approved the manuscript.

\section{Conflicts of Interest}

None declared.

\section{References}

1. Insel TR. The NIMH Research Domain Criteria (RDoC) Project: precision medicine for psychiatry. Am J Psychiatry 2014 Apr;171(4):395-397. [doi: 10.1176/appi.ajp.2014.14020138] [Medline: 24687194]

2. Merikangas KR, Swendsen J, Hickie IB, Cui L, Shou H, Merikangas AK, et al. Real-time Mobile Monitoring of the Dynamic Associations Among Motor Activity, Energy, Mood, and Sleep in Adults With Bipolar Disorder. JAMA Psychiatry 2019 Mar 01;76(2):190-198. [doi: 10.1001/jamapsychiatry.2018.3546] [Medline: 30540352]

3. Broome MR, He Z, Iftikhar M, Eyden J, Marwaha S. Neurobiological and behavioural studies of affective instability in clinical populations: a systematic review. Neurosci Biobehav Rev 2015 Apr;51:243-254. [doi: 10.1016/j.neubiorev.2015.01.021] [Medline: 25662294]

4. Bilderbeck AC, Saunders KEA, Price J, Goodwin GM. Psychiatric assessment of mood instability: qualitative study of patient experience. Br J Psychiatry 2014 Mar;204(3):234-239. [doi: 10.1192/bjp.bp.113.128348] [Medline: 24357573]

5. Judd LL, Schettler PJ, Akiskal HS, Coryell W, Leon AC, Maser JD, et al. Residual symptom recovery from major affective episodes in bipolar disorders and rapid episode relapse/recurrence. Arch Gen Psychiatry 2008 Apr;65(4):386-394. [doi: 10.1001/archpsyc.65.4.386] [Medline: 18391127$]$

6. Bonnín CM, Sánchez-Moreno J, Martínez-Arán A, Solé B, Reinares M, Rosa AR, et al. Subthreshold symptoms in bipolar disorder: impact on neurocognition, quality of life and disability. J Affect Disord 2012 Feb;136(3):650-659. [doi:

10.1016/j.jad.2011.10.012] [Medline: 22051075]

7. Dargél AA, Volant S, Saha S, Etain B, Grant R, Azorin J, FACE-BD collaborators, et al. Activation Levels, Cardiovascular Risk, and Functional Impairment in Remitted Bipolar Patients: Clinical Relevance of a Dimensional Approach. Psychother Psychosom 2019;88(1):45-47. [doi: 10.1159/000493690] [Medline: 30308490]

8. Rosa AR, Franco C, Martínez-Aran A, Sánchez-Moreno J, Reinares M, Salamero M, et al. Functional impairment in patients with remitted bipolar disorder. Psychother Psychosom 2008;77(6):390-392. [doi: 10.1159/000151520] [Medline: 18716425]

9. Dargél AA, Roussel F, Volant S, Etain B, Grant R, Azorin J, FACE-BD Collaborators, et al. Emotional hyper-reactivity and cardiometabolic risk in remitted bipolar patients: a machine learning approach. Acta Psychiatr Scand 2018 Oct;138(4):348-359. [doi: 10.1111/acps.12901] [Medline: 29766490] 
10. Dargél AA, Volant S, Brietzke E, Etain B, Olié E, Azorin J, FACE-BD collaborators. Allostatic load, emotional hyper-reactivity, and functioning in individuals with bipolar disorder. Bipolar Disord 2020 May 16. [doi: 10.1111/bdi.12927] [Medline: $\underline{\text { 32415900] }}$

11. Faurholt-Jepsen M, Frost M, Busk J, Christensen EM, Bardram JE, Vinberg M, et al. Is smartphone-based mood instability associated with stress, quality of life, and functioning in bipolar disorder? Bipolar Disord 2019 Nov;21(7):611-620. [doi: 10.1111/bdi.12796] [Medline: 31081991]

12. Bonsall MB, Wallace-Hadrill SMA, Geddes JR, Goodwin GM, Holmes EA. Nonlinear time-series approaches in characterizing mood stability and mood instability in bipolar disorder. Proc Biol Sci 2012 Mar 07;279(1730):916-924 [FREE Full text] [doi: 10.1098/rspb.2011.1246] [Medline: 21849316]

13. Marwaha S, He Z, Broome M, Singh SP, Scott J, Eyden J, et al. How is affective instability defined and measured? A systematic review. Psychol Med 2013 Sep 27;44(9):1793-1808. [doi: 10.1017/s0033291713002407]

14. Smartphone U. 75 Billion in 2014 Internet. eMarketer. URL: https://www.emarketer.com/Article/ Smartphone-Users-Worldwide-Will-Total-175-Billion-2014/1010536 [accessed 2020-06-24]

15. Frost M, Marcu G, Hansen R, Szaántó K, Bardram J. The MONARCA Self-assessment System: Persuasive Personal Monitoring for Bipolar Patients. Proc 5th Int ICST Conf Pervasive Comput Technol Healthc Internet Dublin, Republic of Ireland: IEEE; 2011 Presented at: Proc 5th Int ICST Conf Pervasive Comput Technol Healthc; 2011; Dublin. [doi: 10.4108/icst.pervasivehealth.2011.246050]

16. Faurholt-Jepsen M, Vinberg M, Christensen EM, Frost M, Bardram J, Kessing LV. Daily electronic self-monitoring of subjective and objective symptoms in bipolar disorder--the MONARCA trial protocol (MONitoring, treAtment and pRediCtion of bipolAr disorder episodes): a randomised controlled single-blind trial. BMJ Open 2013;3(7) [FREE Full text] [doi: 10.1136/bmjopen-2013-003353] [Medline: 23883891]

17. Agarwal V, Zhang L, Zhu J, Fang S, Cheng T, Hong C, et al. Impact of Predicting Health Care Utilization Via Web Search Behavior: A Data-Driven Analysis. J Med Internet Res 2016 Sep 21;18(9):e251 [FREE Full text] [doi: 10.2196/jmir.6240] [Medline: 27655225]

18. World HO. MHealth: new horizons for health through mobile technologies. Geneva: World Health Organization; 2011. URL: https://www.who.int/goe/publications/goe mhealth web.pdf [accessed 2020-01-05]

19. Hanlon P, Daines L, Campbell C, McKinstry B, Weller D, Pinnock H. Telehealth Interventions to Support Self-Management of Long-Term Conditions: A Systematic Metareview of Diabetes, Heart Failure, Asthma, Chronic Obstructive Pulmonary Disease, and Cancer. J Med Internet Res 2017 May 17;19(5):e172 [FREE Full text] [doi: 10.2196/jmir.6688] [Medline: 28526671]

20. Faurholt-Jepsen M, Frost M, Vinberg M, Christensen EM, Bardram JE, Kessing LV. Smartphone data as objective measures of bipolar disorder symptoms. Psychiatry Res 2014 Jun 30;217(1-2):124-127. [doi: 10.1016/j.psychres.2014.03.009] [Medline: 24679993]

21. Richards D, Richardson T. Computer-based psychological treatments for depression: a systematic review and meta-analysis. Clin Psychol Rev 2012 Jun;32(4):329-342. [doi: 10.1016/j.cpr.2012.02.004] [Medline: 22466510]

22. Berry N, Lobban F, Emsley R, Bucci S. Acceptability of Interventions Delivered Online and Through Mobile Phones for People Who Experience Severe Mental Health Problems: A Systematic Review. J Med Internet Res 2016 May 31;18(5):e121 [FREE Full text] [doi: 10.2196/jmir.5250] [Medline: 27245693]

23. Riper H, Spek V, Boon B, Conijn B, Kramer J, Martin-Abello K, et al. Effectiveness of E-self-help interventions for curbing adult problem drinking: a meta-analysis. J Med Internet Res 2011;13(2):e42 [FREE Full text] [doi: 10.2196/jmir.1691] [Medline: 21719411]

24. Donker T, Petrie K, Proudfoot J, Clarke J, Birch M, Christensen H. Smartphones for smarter delivery of mental health programs: a systematic review. J Med Internet Res 2013;15(11):e247 [FREE Full text] [doi: 10.2196/jmir.2791] [Medline: 24240579]

25. Depp CA, Ceglowski J, Wang VC, Yaghouti F, Mausbach BT, Thompson WK, et al. Augmenting psychoeducation with a mobile intervention for bipolar disorder: a randomized controlled trial. J Affect Disord 2015 Mar 15;174:23-30. [doi: 10.1016/j.jad.2014.10.053] [Medline: 25479050]

26. Mayo-Wilson E, Montgomery P. Media-delivered cognitive behavioural therapy and behavioural therapy (self-help) for anxiety disorders in adults. Cochrane Database Syst Rev 2013;9:CD005330. [doi: 10.1002/14651858.CD005330.pub4] [Medline: 24018460]

27. Lauder S, Chester A, Castle D, Dodd S, Gliddon E, Berk L, et al. A randomized head to head trial of MoodSwings.net.au: an Internet based self-help program for bipolar disorder. J Affect Disord 2015 Jan 15;171:13-21. [doi:

10.1016/i.jad.2014.08.008] [Medline: 25282145]

28. Proudfoot J, Parker G, Manicavasagar V, Hadzi-Pavlovic D, Whitton A, Nicholas J, et al. Effects of adjunctive peer support on perceptions of illness control and understanding in an online psychoeducation program for bipolar disorder: a randomised controlled trial. J Affect Disord 2012 Dec 15;142(1-3):98-105. [doi: 10.1016/j.jad.2012.04.007] [Medline: 22858215]

29. Barnes CW, Hadzi-Pavlovic D, Wilhelm K, Mitchell PB. A web-based preventive intervention program for bipolar disorder: outcome of a 12-months randomized controlled trial. J Affect Disord 2015 Mar 15;174:485-492. [doi:

10.1016/j.jad.2014.11.038] [Medline: 25554993] 
30. Duffy A, Keown-Stoneman CD, Goodday SM, Saunders K, Horrocks J, Grof P, et al. Daily and weekly mood ratings using a remote capture method in high-risk offspring of bipolar parents: Compliance and symptom monitoring. Bipolar Disord 2019 Mar;21(2):159-167. [doi: 10.1111/bdi.12721] [Medline: 30422376]

31. Wenze SJ, Armey MF, Miller IW. Feasibility and Acceptability of a Mobile Intervention to Improve Treatment Adherence in Bipolar Disorder: A Pilot Study. Behav Modif 2014 Jan 8;38(4):497-515. [doi: 10.1177/0145445513518421] [Medline: 24402464]

32. McKnight RF, Bilderbeck AC, Miklowitz DJ, Hinds C, Goodwin GM, Geddes JR. Longitudinal mood monitoring in bipolar disorder: Course of illness as revealed through a short messaging service. J Affect Disord 2017 Dec 01;223:139-145. [doi: 10.1016/j.jad.2017.07.029] [Medline: 28753472]

33. Hidalgo-Mazzei D, Reinares M, Mateu A, Nikolova V, Bonnín CDM, Samalin L, et al. OpenSIMPLe: A real-world implementation feasibility study of a smartphone-based psychoeducation programme for bipolar disorder. J Affect Disord 2018 Dec 01;241:436-445. [doi: 10.1016/j.jad.2018.08.048] [Medline: $\underline{30145515}$ ]

34. Saunders KEA, Bilderbeck AC, Panchal P, Atkinson LZ, Geddes JR, Goodwin GM. Experiences of remote mood and activity monitoring in bipolar disorder: A qualitative study. Eur Psychiatry 2017 Mar;41:115-121. [doi: 10.1016/j.eurpsy.2016.11.005] [Medline: 28135594]

35. Young AS, Cohen AN, Niv N, Nowlin-Finch N, Oberman RS, Olmos-Ochoa TT, et al. Mobile Phone and Smartphone Use by People With Serious Mental Illness. Psychiatr Serv 2020 Mar 01;71(3):280-283. [doi: 10.1176/appi.ps.201900203] [Medline: $\underline{31744429]}$

36. Whybrow PC, Grof P, Gyulai L, Rasgon N, Glenn T, Bauer M. The electronic assessment of the longitudinal course of bipolar disorder: the ChronoRecord software. Pharmacopsychiatry 2003 Nov;36 Suppl 3:S244-S249. [doi: 10.1055/s-2003-45137] [Medline: 14677086]

37. Tsanas A, Saunders KEA, Bilderbeck AC, Palmius N, Osipov M, Clifford GD, et al. Daily longitudinal self-monitoring of mood variability in bipolar disorder and borderline personality disorder. J Affect Disord 2016 Nov 15;205:225-233 [FREE Full text] [doi: 10.1016/j.jad.2016.06.065] [Medline: $\underline{27449555]}$

38. True Colours. NHS. URL: https://ouh.truecolours.nhs.uk/ibd/en/ [accessed 2020-01-05]

39. Faurholt-Jepsen M, Geddes JR, Goodwin GM, Bauer M, Duffy A, Vedel Kessing L, et al. Reporting guidelines on remotely collected electronic mood data in mood disorder (eMOOD)-recommendations. Transl Psychiatry 2019 Jun 07;9(1):162 [FREE Full text] [doi: 10.1038/s41398-019-0484-8] [Medline: 31175283]

40. Goldbeter A. A model for the dynamics of bipolar disorders. Prog Biophys Mol Biol 2011 Mar;105(1-2):119-127. [doi: 10.1016/j.pbiomolbio.2010.11.007] [Medline: 21115030]

41. Torous J, Firth J, Mueller N, Onnela JP, Baker JT. Methodology and Reporting of Mobile Heath and Smartphone Application Studies for Schizophrenia. Harv Rev Psychiatry 2017;25(3):146-154 [FREE Full text] [doi: 10.1097/HRP.0000000000000133] [Medline: 28234658]

42. Platon. Oeuvres complètes. Paris: Flammarion; 2008:978-972.

43. Montgomery SA, Asberg M. A new depression scale designed to be sensitive to change. Br J Psychiatry 1979 Apr;134:382-389. [doi: 10.1192/bjp.134.4.382] [Medline: 444788]

44. Young RC, Biggs JT, Ziegler VE, Meyer DA. A rating scale for mania: reliability, validity and sensitivity. Br J Psychiatry 1978 Nov;133:429-435. [doi: 10.1192/bjp.133.5.429] [Medline: 728692]

45. Rush AJ, Trivedi MH, Ibrahim HM, Carmody TJ, Arnow B, Klein DN, et al. The 16-Item Quick Inventory of Depressive Symptomatology (QIDS), clinician rating (QIDS-C), and self-report (QIDS-SR): a psychometric evaluation in patients with chronic major depression. Biol Psychiatry 2003 Sep 01;54(5):573-583. [doi: 10.1016/s0006-3223(02)01866-8] [Medline: 12946886]

46. Altman EG, Hedeker D, Peterson JL, Davis JM. The Altman Self-Rating Mania Scale. Biol Psychiatry 1997 Nov 15;42(10):948-955. [doi: 10.1016/S0006-3223(96)00548-3] [Medline: 9359982]

47. Buysse DJ, Reynolds CF, Monk TH, Berman SR, Kupfer DJ. The Pittsburgh Sleep Quality Index: a new instrument for psychiatric practice and research. Psychiatry Res 1989 May;28(2):193-213. [doi: 10.1016/0165-1781(89)90047-4] [Medline: 2748771]

48. Henry C, M'Bailara K, Mathieu F, Poinsot R, Falissard B. Construction and validation of a dimensional scale exploring mood disorders: MAThyS (Multidimensional Assessment of Thymic States). BMC Psychiatry 2008 Sep 19;8(1). [doi: $10.1186 / 1471-244 \mathrm{x}-8-82]$

49. Research Electronic Data Capture, NIH. URL: https://www.project-redcap.org/ [accessed 2020-01-08]

50. Toi Même: a Mobile System for Measuring Bipolar Illness. ClinicalTrials.gov. URL: https://clinicaltrials.gov/ct2/show/ NCT03508427 [accessed 2020-01-09]

51. Thompson K, Kulkarni J, Sergejew A. Reliability and validity of a new Medication Adherence Rating Scale (MARS) for the psychoses. Schizophrenia Research 2000 May;42(3):241-247. [doi: 10.1016/s0920-9964(99)00130-9]

52. Kieser M, Friede T. Re-calculating the sample size in internal pilot study designs with control of the type I error rate. Statist. Med 2000 Apr 15;19(7):901-911. [doi: 10.1002/(sici)1097-0258(20000415)19:7<901::aid-sim405>3.0.co;2-1]

53. Greiner M, Pfeiffer D, Smith R. Principles and practical application of the receiver-operating characteristic analysis for diagnostic tests. Preventive Veterinary Medicine 2000 May;45(1-2):23-41. [doi: 10.1016/S0167-5877(00)00115-X] 
54. Hidalgo-Mazzei D, Murru A, Reinares M, Vieta E, Colom F. Big Data in mental health: a challenging fragmented future. World Psychiatry 2016 Jun;15(2):186-187 [FREE Full text] [doi: 10.1002/wps.20307] [Medline: 27265716]

55. Laney D. Controlling Data Volume, Velocity, and Variety Internet. 3D Data Management: METAGroup; 2001 Feb 06. URL: https://blogs.gartner.com/doug-laney/files/2012/01/ ad949-3D-Data-Management-Controlling-Data-Volume-Velocity-and-Variety.pdf [accessed 2020-01-18]

56. Torous J, Baker JT. Why Psychiatry Needs Data Science and Data Science Needs Psychiatry: Connecting With Technology. JAMA Psychiatry 2016 Jan;73(1):3-4. [doi: 10.1001/jamapsychiatry.2015.2622] [Medline: 26676879]

57. Monteith S, Glenn T, Geddes J, Bauer M. Big data are coming to psychiatry: a general introduction. Int J Bipolar Disord 2015 Dec;3(1):21 [FREE Full text] [doi: 10.1186/s40345-015-0038-9] [Medline: 26440506]

58. McIntyre RS, Cha DS, Jerrell JM, Swardfager W, Kim RD, Costa LG, et al. Advancing biomarker research: utilizing 'Big Data' approaches for the characterization and prevention of bipolar disorder. Bipolar Disord 2014 Aug;16(5):531-547. [doi: 10.1111/bdi.12162] [Medline: 24330342]

\author{
Abbreviations \\ AMoSS: Automated Monitoring of Symptoms Severity \\ CTPJ-TB: Centre Thérapeutique de Jour-Troubles Bipolaires \\ DSM-5: Diagnostic and Statistical Manual of Mental Disorders, 5th edition \\ FAST: Functioning Assessment Short Test \\ MADRS: Montgomery-Åsberg Depression Rating Scale \\ mHealth: mobile health \\ MONARCA: MONitoring, treAtment and pRediCtion of bipolAr Disorder Episodes \\ PCA: principal component analysis \\ QIDS-SR: Quick Inventory of Depressive Symptomatology Self-Report \\ REDCap: Research Electronic Data Capture \\ YMRS: Young Mania Rating Scale
}

\author{
Edited by G Eysenbach; submitted 20.03.20; peer-reviewed by J Lee, L Zhang, F Lamers, V Bremer; comments to author 12.06.20; \\ revised version received 24.06.20; accepted 25.06.20; published 18.08.20 \\ Please cite as: \\ Dargél AA, Mosconi E, Masson M, Plaze M, Taieb F, Von Platen C, Buivan TP, Pouleriguen G, Sanchez M, Fournier S, Lledo PM, \\ Henry $C$ \\ Toi Même, a Mobile Health Platform for Measuring Bipolar Illness Activity: Protocol for a Feasibility Study \\ JMIR Res Protoc 2020;9(8):e18818 \\ URL: http://www.researchprotocols.org/2020/8/e18818/ \\ doi: $10.2196 / 18818$ \\ PMID: 32638703
}

(C)Aroldo A Dargél, Elise Mosconi, Marc Masson, Marion Plaze, Fabien Taieb, Cassandra Von Platen, Tan Phuc Buivan, Guillaume Pouleriguen, Marie Sanchez, Stéphane Fournier, Pierre-Marie Lledo, Chantal Henry. Originally published in JMIR Research Protocols (http://www.researchprotocols.org), 18.08.2020. This is an open-access article distributed under the terms of the Creative Commons Attribution License (https://creativecommons.org/licenses/by/4.0/), which permits unrestricted use, distribution, and reproduction in any medium, provided the original work, first published in JMIR Research Protocols, is properly cited. The complete bibliographic information, a link to the original publication on http://www.researchprotocols.org, as well as this copyright and license information must be included. 